\title{
Myocardial Infarction Associated With Psoriasis
}

\author{
Luxhman Gunaseelan, $\mathrm{MD}^{1}$ - Sumairaa Ahmed, $\mathrm{BSc}^{2}$ - Arooj Malik, MD $\mathrm{MD}^{3}$ Muhammad Akhter Hamid, MD, DCH, MRCPI
}

\begin{abstract}
A 54-year-old man presented to our emergency department (ED) with a 2-hour history of shortness of breath and dull left-sided chest pain that radiated to the left shoulder and jaw. The shortness of breath and chest pain were exacerbated with activity but were also present at rest. The patient also reported weakness, dizziness, nausea, and one episode of emesis.
\end{abstract}

\section{History}

He had an 11-year history of severe plaque psoriasis and 5-year history of psoriatic arthritis. The patient had no history of hypertension or hypercholesterolemia. An annual checkup 3 weeks earlier had revealed no abnormalities apart from severe psoriasis; his total cholesterol, low-density lipoprotein cholesterol, and high-density lipoprotein cholesterol levels, as well as blood pressure, were within normal limits.

The patient had no family history of coronary artery disease, psoriasis, hyper- tension, or dyslipidemia. The patient was a lifetime nonsmoker and denied alcohol consumption. He did not take prescribed medications, and his only regular overthe-counter medications were as-needed ibuprofen for psoriatic arthritis and topical emollients for psoriatic skin lesions.

He followed a strict vegan diet, which he felt lessened the severity of the pruritis, rash, and arthritis associated with psoriasis.

\section{Physical examination}

A general examination revealed a diaphoretic man under visible distress. A cardiovascular examination revealed normal cardiac sounds, a normal blood pressure of $172 / 94 \mathrm{~mm} \mathrm{Hg}$, and a normal heart rate of 113 beats $/ \mathrm{min}$. No chest-wall tenderness was noted. A pulmonary examination revealed an elevated respiratory rate of 22 breaths/min. An abdominal examination revealed normal bowel sounds and no tenderness or rebound tenderness. A skin examination revealed

\section{AFFILIATIONS:}

${ }^{1}$ Clinical Research Coordinator, Scarborough Health Network, Toronto, Canada

${ }^{2}$ Bachelors of Science Student, McMaster University, Hamilton, Ontario, Canada

${ }^{3}$ Medical Student, Xavier School of Medicine, Oranjestad, Aruba

${ }^{4}$ Assistant Professor of Pediatrics, University of Toronto, Ontario, Canada

\section{CITATION:}

Gunaseelan L, Ahmed S, Malik A, Akhter Hamid, M. Myocardial infarction associated with psoriasis. Consultant. Published online February 2, 2022. doi:10.25270/con.2022.02.00001

Received September 5, 2021. Accepted October 12, 2021.

\section{DISCLOSURES:}

The authors report no relevant financial relationships.

\section{CORRESPONDENCE:}

Luxhman Gunaseelan, MD, Scarborough Health Network, 3030 Lawrence Ave East, Suite 314, Scarborough, ON M1P 2T7, Canada (v.lgunaseelan@shn.ca)
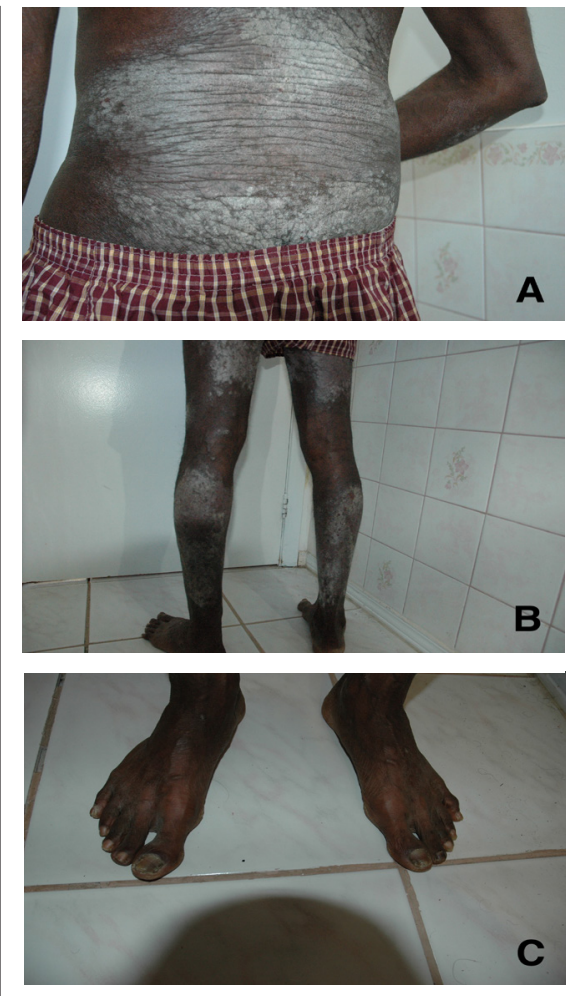

Figure 1. The patient's skin on his lower back showed well-circumscribed, thickened, scaly, silvery-white plaques indicative of severe psoriasis (A). His extensor and flexural surfaces of the lower limbs showed well-defined, scaly, silvery-white plaques indicative of severe psoriasis (B). The patient's feet showed hypertrophy and pitted surface of the nails, indicative of psoriatic nail changes (C).

well-defined, scaly, silvery-white plaques on the extensor and flexural surfaces of the forearms, elbows, lower back, abdomen, and most of the lower limbs (Figure 1). The patient's Psoriasis Area Severity Index score was 33.8, indicating very severe psoriasis. Examination of the nails revealed dystrophy with extensive pitting of the toenails (Figure 1). Decreased range of motion was noted and pain was 


\section{Table. Summary of Troponin Values Over the Hospital Course}

DATE AND TIME
11-Sep 1:31 PM
11-Sep 11:00 PM
12-Sep 6:00 AM

elicited on passive and active range of movement of the bilateral metacarpophalangeal joints, lower back, patellar joints, and hip.

\section{Diagnostic testing}

Results from laboratory testing conducted upon admission to the hospital revealed an elevated troponin level of 1.22 $\mathrm{ng} / \mathrm{mL}$. Ten hours later, the patient's troponin level increased to $41.13 \mathrm{ng} / \mathrm{mL}$ (Table).

An electrocardiogram revealed ST segment elevation in leads $1, A v L$, and V2 to $\mathrm{V} 5$ and peaked $\mathrm{T}$ waves in leads $\mathrm{V} 2$ to V4, indicating anterolateral ST-elevation myocardial infarction. A coronary angiography was then performed, results of which revealed occlusion of the posterior mid-left anterior descending coronary artery (Figure 2). A bare metal stent was placed at the occlusion. Door-to-balloon time was 78 minutes.

Three days later, the patient's troponin levels decreased to $1.60 \mathrm{ng} / \mathrm{mL}$. The patient was prescribed clopidogrel, $75 \mathrm{mg}$; bisoprolol, $5 \mathrm{mg}$; ramipril, $2.5 \mathrm{mg}$; atorvastatin, $40 \mathrm{mg}$; and aspirin, $81 \mathrm{mg}$ and was discharged home once he was stable.

\section{Discussion}

Psoriasis is a noncontagious inflammatory skin condition affecting approximately $2 \%$ of the global population. ${ }^{1}$ It is characterized by pruritic, erythematous, and scaly rashes varying in size, most commonly affecting the forearms, shins, navel area, and scalp.' Due to its inflammatory nature and the possibility of shared inflammatory pathways, psoriasis may lead to dysfunction of the cardiovascular, pulmonary, renal, and endocrine systems. Our patient had experienced a myocardial infarction prematurely at the

\section{TROPONIN I (NG/ML)}

1.22

41.13

22.75

1.60

age of 54 years despite having a calculated 10-year atherosclerotic cardiovascular disease risk of only $4.9 \%$. He had no other cardiovascular risk factors-including hypertension, dyslipidemia, or diabetesor any family history of cardiovascular disease. He had a Psoriasis Area Severity Index score of 33.8, indicating very severe psoriasis ${ }^{2}$, a systemic inflammatory condition that was at one time thought to only be limited to the skin and joints, but it is now known to be associated with various systemic comorbidities. Among these is a possible association with increased risk of severe vascular events such as myocardial infarction.

A cross-sectional study conducted in 2015 included 4185 patients with psoriasis and a prospective cohort of 1.8 million German Health Insurance beneficiaries. ${ }^{3}$ Findings revealed a significant association between psoriasis and myocardial infarction (odds ratio [OR], 2.26; $95 \% \mathrm{Cl}$, 1.03-4.96) and type 2 diabetes (OR, 2.36; $95 \% \mathrm{Cl}, 1.26-4.41))^{3}$ The researchers also examined the genetic architecture of psoriasis and cardiometabolic disease but found no strong associations between polymorphisms underlying psoriasis and coronary artery disease, indicating that the 2 diseases are genetically distinct. ${ }^{3}$

The association between psoriasis and cardiovascular disease may likely be due to the shared inflammatory pathways between them. Psoriasis is an immune-mediated skin disease characterized by abnormal proliferation and differentiation of keratinocytes, which release inflammatory cytokines. This leads not only to inflammation of the skin, which worsens the dermatological manifestations of the psoriasis, but also to vascular remodeling, which can contribute to cardiovascular disease. ${ }^{4}$ In susceptible populations, psoriasis starts with trauma to the keratinocytes in the epithelium. Injured keratinocytes release complexes of host DNA and cathelicidin to activate antigen-presenting plasmacytoid dendritic cells. This activation stimulates T helper 1 (Th1) and T helper 17 (Th17) lymphocytes and produces tumor necrosis factor- $\alpha$ (TNF- $\alpha$ ), interleukin-12 (IL-12), and IL-23. IL-23 stimulates production and activation of Th17 cells, $\mathrm{T}$ cells that release IL-17. IL-17 upregulates the proliferation of keratinocytes and downregulates the differentiation of keratinocytes, thereby leading to the characteristic scaly pruritic plaques associated with psoriasis and further inflammatory cytokine release. ${ }^{4}$ These inflammatory cytokines and Th1 and Th17 lymphocytes are commonly found in both unstable atherosclerotic lesions in patients with cardiovascular disease and the lesions seen in patients with psoriasis, suggesting shared inflammatory pathways linking psoriasis and atherosclerotic disease. ${ }^{5}$

According to Pina and colleagues, inflammatory molecules such as TNF- $\alpha$ promote vascular remodeling, leading to endothelial dysfunction and arterial stiffness associated with cardiovascular disease. ${ }^{6}$ Pulse wave velocity (PWV) and flow-mediated vasodilation (FMV) assess vessel endothelial function and can be considered as surrogate markers for atherosclerotic disease. ${ }^{6}$ Patients with chronic psoriasis and psoriatic arthritis, even without other cardiovascular risk factors such as hypertension, dyslipidemia, or diabetes, have decreased FMV and increased PWV-changes characteristic of a stiff atherosclerotic vessel. Pina and colleagues reported that the use of anti-TNF- $\alpha$ therapy (adalimumab) increased $F M V \%$ from $6.19 \pm 2.44 \%$ at the initiation of adalimumab to $7.46 \pm 2.43 \%$ after 6 months $(P=.008)$ and decreased PWV levels from $6.28 \pm 1.04 \mathrm{~m} / \mathrm{s}$ at the initiation of adalimumab to $5.69 \pm 1.31$ $\mathrm{m} / \mathrm{s}$ after 6 months $(P=.03){ }^{6}$

Chronic systemic inflammation associated with psoriasis may also contribute 


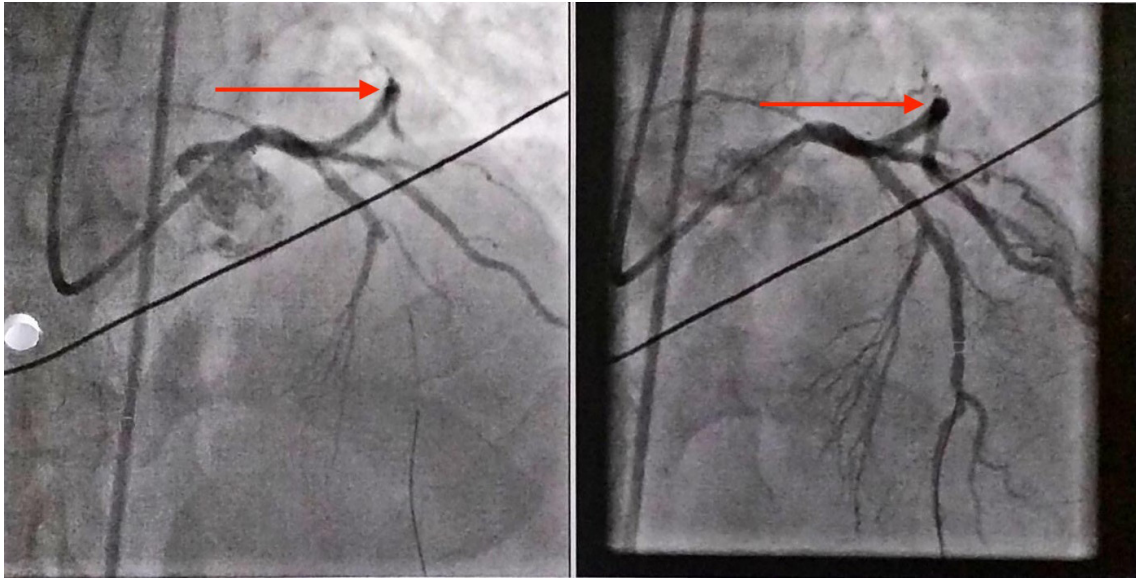

Figure 2. A coronary angiogram was performed shortly after admission. Note the occlusion of the posterior mid-left anterior descending coronary artery.

to cardiovascular disease through the formation of microparticles, membrane vesicles with nucleic acids, and inflammatory mediators such as IL-1, Cluster of Differentiation 40 ligands, and intercellular adhesion molecule 1 (ICAM 1). These molecules contribute to vascular inflammation, thrombosis, and accelerated atherogenesis. ${ }^{7}$

Patients with psoriasis have an increased risk of cardiovascular disease mortality independent of traditional cardiovascular disease risk factors. $A$ hospital-based multivariate analysis conducted by Shiba and colleagues analyzed the prevalence of coronary heart disease, hypertension, dyslipidemia, diabetes, and psoriasis in 113,065 patients. ${ }^{8}$ Overall, $14.5 \%$ of participants had hypertension, $10.2 \%$ had diabetes, $8.1 \%$ had dyslipidemia, $4.5 \%$ had coronary heart disease, and $1.1 \%$ had psoriasis vulgaris. They found that psoriasis was an independent variable associated with all of these conditions. ${ }^{8}$ This may explain why our patient, who had psoriasis but no other cardiovascular disease risk factors, had experienced a myocardial infarction.

In addition to the possibility that psoriasis may be an independent risk factor for cardiovascular disease, psoriasis can also lead to the development of metabolic syndrome, a cluster of conditions that can increase heart disease risk. These include type 2 diabetes, hypertriglycer- idemia, low high-density lipoprotein cholesterol, increased waist circumference, and hypertension. ${ }^{9}$ Chronic systemic inflammation from psoriasis leads to inflammation of adipose tissue, which releases adipokines, a family of inflammatory mediators, into the circulation. Adipocyte-derived retinol-binding protein 4, a type of adipokine, may lead to insulin resistance and is increased in patients with both type 2 diabetes and psoriasis. Leptin, another type of adipokine, promotes activation of $T$ cells and monocytes, further promoting systemic inflammation. Resistin is another adipokine found in patients with psoriasis that promotes atherosclerosis. ${ }^{5}$

Psoriasis is also associated with an increased prevalence and incidence of type 2 diabetes and hypertension. A series of meta-analyses and a systematic review compared the prevalence and incidence of diabetes and hypertension in patients with psoriasis with the incidence of these conditions in healthy control groups. ${ }^{10,11}$ The odds ratio for diabetes was 1.97 (95\% $\mathrm{Cl}, 1.48-2.62)$ and for hypertension was 1.49 (95\% Cl, 1.20-1.86) for those with severe psoriasis, compared with healthy controls. ${ }^{10,11}$

Psoriasis is also associated with an increased prevalence and incidence of dyslipidemia, as revealed by Dreiher and colleagues, ${ }^{12}$ who conducted a cross-sectional population study that showed dys- lipidemia was significantly higher among patients with psoriasis (OR, 1.48; $95 \% \mathrm{Cl}$, $1.40-1.55)$ than those without psoriasis. ${ }^{12}$

Despite these findings, it is wise to consider psoriasis-associated inflammatory pathways when devising strategies to reduce the risk for cardiovascular events in patients with psoriasis. Such strategies could involve treatment focused on the inflammatory roots of psoriasis, as well as aggressively addressing any other risk factors for cardiovascular disease that might be present, such as metabolic syndrome. A retrospective cohort study conducted at Kaiser Permanente Southern California investigated the link between TNF-inhibitor therapy and myocardial infarction risk in patients with psoriasis and no history of previous myocardial infarction. ${ }^{13}$ Patients using a TNF inhibitor experienced a significantly decreased risk of myocardial infarction (adjusted hazard ratio, 0.50; $95 \% \mathrm{Cl}$, 0.32-0.79) compared with patients using topical medication (ie, coal tar, corticosteroid creams). ${ }^{13}$

\section{Patient outcome}

At the time of his emergency department visit, our patient was not taking any medications for his severe psoriasis. We recommended that he discuss the benefits of taking methotrexate and biologics such as adalimumab with his primary care physician to help reduce his risk for future cardiovascular events. As psoriasis is strongly associated with features of metabolic syndrome such as hypertension, dyslipidemia, and type 2 diabetes, patients like ours should be monitored and treated for these conditions if present.

\section{Conclusions}

It is important to appreciate the systemic manifestations of psoriasis. It is not a disease only affecting the skin and joints, but because of shared inflammatory pathways between these systems, psoriasis may be associated with cardiovascular and metabolic disease as well. 


\section{References}

1. Boehncke WH, Schön MP. Psoriasis. Lancet. 2015;386(9997):983-994. https://doi. org/10.1016/s0140-6736(14)61909-7

2. Schmitt J, Wozel G. The psoriasis area and severity index is the adequate criterion to define severity in chronic plaque-type psoriasis. Dermatology, 2005;210(3):194-199. https://doi.org/10.1159/000083509

3. Koch M, Baurecht $\mathrm{H}$, Ried JS, et al. Psoriasis and cardiometabolic traits: modest association but distinct genetic architectures. J Invest Dermatol. 2015;135(5):1283-1293. https://doi.org/10.1038/jid.2015.8

4. Furue M, Tsuji G, Chiba T, Kadono T. Cardiovascular and metabolic diseases comorbid with psoriasis: beyond the skin. Intern Med. 2017;56(13):1613-1619. https://doi. org/10.2169/internalmedicine.56.8209

5. Ryan C, Kirby B. Psoriasis is a systemic disease with multiple cardiovascular and metabolic comorbidities. Dermatol Clin. 2015;33(1):41-55. https://doi.org/10.1016/j. det.2014.09.004

6. Pina T, Corrales A, Lopez-Mejias R, et al. Anti-tumor necrosis factor-alpha therapy improves endothelial function and arterial stiffness in patients with moderate to severe psoriasis: A 6-month prospective study. J Dermatol. 2016;43(11):1267-1272. https://doi.org/10.1111/1346-8138.13398

7. Takeshita J, Mohler ER, Krishnamoorthy P, et al. Endothelial cell-, platelet-, and monocyte/macrophage-derived microparticles are elevated in psoriasis beyond cardiometabolic risk factors. J Am Heart Assoc. 2014;3(1):e000507. https://doi.org/10.1161/ jaha.113.000507

8. Shiba M, Kato T, Funasako M, et al.
Association between psoriasis vulgaris and coronary heart disease in a hospital-based population in Japan. PLoS One. 2016;11(2):e0149316. https://doi.org/10.1371/ journal.pone.0149316

9. Alberti KG, Eckel RH, Grundy SM, et al. Harmonizing the metabolic syndrome: a joint interim statement of the International Diabetes Federation Task Force on Epidemiology and Prevention; National Heart, Lung, and Blood Institute; American Heart Association; World Heart Federation; International Atherosclerosis Society; and International Association for the Study of Obesity. Circulation. 2009;120(16):16401645. https://doi.org/10.1161/circulationaha.109.192644

10. Armstrong AW, Harskamp CT, Armstrong EJ. Psoriasis and the risk of diabetes mellitus: a systematic review and meta-analysis. JAMA Dermatol. 2013;149(1):84-91. https:// doi.org/10.1001/2013.jamadermatol.406

11. Armstrong AW, Harskamp CT, Armstrong EJ. The association between psoriasis and hypertension: a systematic review and meta-analysis of observational studies. $J$ Hypertens, 2013;31(3):433-443. https://doi. org/10.1097/hjh.0b013e32835bcce1

12. Dreiher J, Weitzman D, Davidovici B, Shapiro J, Cohen AD. Psoriasis and dyslipidaemia: a population-based study. Acta Derm Venereol. 2008;88(6):561-565. https://doi. org/10.2340/00015555-0510

13. Wu JJ, Poon KY, Channual JC, Shen AY. Association between tumor necrosis factor inhibitor therapy and myocardial infarction risk in patients with psoriasis. Arch Dermatol. 2012;148(11):1244-1250. https://doi. org/10.1001/archdermatol.2012.2502 